\title{
Internationales Team forscht an kohlenstoffbasierten Materialien
}

Die BASF und das Max-Planck-Institut für Polymerforschung (MPI-P) haben kürzlich ihre gemeinsame Forschungsund Entwicklungsplattform, das Carbon Materials Innovation Center (CMIC), auf dem BASF-Firmengelände in Ludwigshafen eröffnet.

— ine multidisziplinäre Arbeitsgruppe — wird die wissenschaftlichen Grundlagen und Anwendungsmöglichkeiten innovativer Materialien auf Kohlenstoffbasis erforschen. Das internationale zwölfköpfige Team setzt sich aus Chemi- kern, Physikern und Materialwissenschaftlern zusammen. In dem 200 Quadratmeter großen Labor werden unter anderem neue Materialien synthetisiert und charakterisiert und deren Anwendungsmöglichkeiten im Energie- und Elektronikbereich überprüft. Die Investitionssumme für die gemeinsame Forschungs- und Entwicklungsplattform beträgt €10 Millionen. Die Kooperation ist zunächst für drei Jahre geplant.

„Wir stehen am Anfang einer neuen Querschnittstechnologie, die zahlreiche Anwendungen revolutionieren und In- novationen ermöglichen wird. Das Rennen um die zukünftigen Einsatzmöglichkeiten von kohlenstoffbasierten Materialien wie Graphen ist in vollem Gange und wir möchten ganz vorne mit dabei sein, wenn es darum geht, die Möglichkeiten zu nutzen“, sagt Dr. Andreas Kreimeyer, Vorstandsmitglied der BASF und Sprecher der Forschung, bei der Eröffnung des Labors.

Das CMIC ist die erste Forschungsplattform, die die BASF gemeinsam mit einem wissenschaftlichen Partner an einem BASF-Standort betreibt.

\section{Reverdia und Helm vermarkten gemeinsam biobasierte Bernsteinsäure}

Peverdia, ein Joint Venture zwischen R DSM und Roquette, und Helm haben einen Kooperationsvertrag über die Vermarktung und Distribution der von Reverdia produzierten biobasierten Bernsteinsäure in Europa unterzeichnet. Diese Bernsteinsäure mit der Markenbezeichnung Biosuccinium dient als wirtschaftliche und ökologische Alternative zur erdölbasierten Adipinsäure und wird u.a. für die Produktion von Klebstoffen, Kühlmitteln, Tensiden, Korrosionsschutzmitteln, Schmiermitteln, Filmentwicklungsflüssigkeiten, Herbiziden, Lötpasten, Enteisungsmitteln, Polyurethanen, Lackharzen, phthalatfreien Weichma-

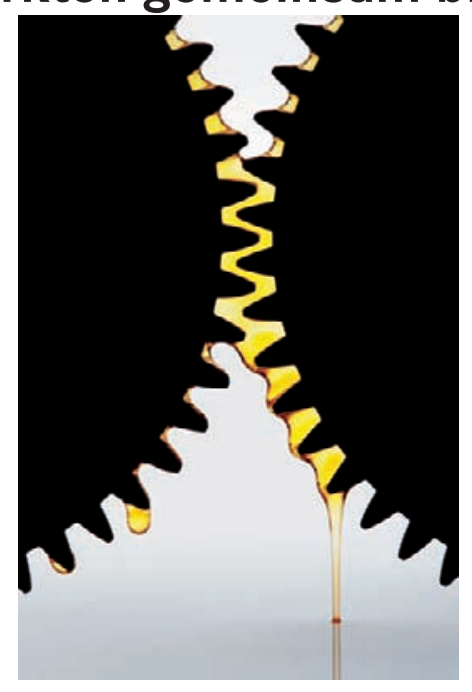

chern und Polybutylsuccinat (PBS) eingesetzt.

Die Herstellung der Bernsteinsäure erfolgt seit Anfang 2010 in Lestrem, Frankreich mit einer Kapazität von 300 Tonnen/ Jahr auf einer Demoanlage nach einem geschützten Verfahren durch Fermentation von Hefe. Ende 2012 wird Reverdia die Produktion in Cassano Spinola, Italien, aufnehmen.

Biobasierte Bernsteinsäure wird u. a. für die Herstellung von Schmiermitteln, aber auch Klebstoffen und vielen anderen Produkten eingesetzt.

(Quelle: Helm: REVPROO2)

\section{Europäische Methode zur Prüfung von VOC-Emissionen harmonisiert}

Ein Information der Prüflabors Eurofins Product Testing produkte-Verordnung die harmonisierte europäische Prüfmethode für VOC-Emissionen von der zuständigen Arbeitsgruppe beim CEN verabschiedet.

Laut Zeitplan sollen in ein bis zwei Jahren VOC-Prüfungen für das CE-Zeichen mit dieser Methode durchgeführt werden. Darüber hinaus ist davon auszugehen, dass zukünftig diverse nationale Gütezeichen für emissionsarme Produkte auf der Basis entsprechend harmonisierter Prüfmethoden verliehen werden.

Emissionsprüfungen werden an verschiedenen Produkten vorgenommen, um die Übereinstimmung mit gesetzlichen An- forderungen oder mit freiwilligen Ökolabels zu dokumentieren - beispielsweise für Bauprodukte, Möbel und Ausbaukomponenten für den Innenraum von KFZ. Die Reproduzierbarkeit dieser Prüfungen von Labor zu Labor war bisher jedoch nicht immer zufriedenstellend.

Um die Qualität zu verbessern, sind im jetzt verabschiedeten Standard u.a. genau definierte Prüfkammer-Parameter und die zulässigen Abweichungen festgelegt. Die wichtigste Veränderung aber ist, dass die Berechnung der Emissionen für alle Testergebnisse nach einem europäischen Referenzverfahren erfolgen soll.

Eurofins bietet zu diesem Thema eine Serie von Seminaren an. Weitere Infos: www.eurofins.com/galten 